\title{
FUENTES PRIMARIAS PARA EL ESTUDIO DEL APROVECHAMIENTO DE LOS RECURSOS HIDRÁULICOS EN MÉXICO. EL AHCRG
}

\author{
María Teresa Pacho Rodríguez \\ Gabriel Rivera García *
}

INTRODUCCIÓN

I a necesidad de recuperar archivos históricos y conformar acervos que proporcionen sólidas bases documentales a la investigación científica es indudable e imperiosa. Todo esfuerzo que se emprenda para satisfacerla se hace también para propiciar descubrimientos, constatar hipótesis, identificar datos y verificar fechas.

Uno de los proyectos recientes de esta índole es la conformación del Archivo Histórico de la Comisión del Río Grijalva (AHCRG), que forma parte de un amplio programa de la Comisión Nacional del Agua para dar a conocer al público sus antecedentes y su desarrollo.

La Comisión del Río Grijalva dependía de la desaparecida Secretaría de Agricultura y Recursos Hidráulicos; fue constituida en 1951 y concluyó sus actividades en 1985. En este periodo desarrolló una labor que amerita cuidadosos estudios para que sus fines y acciones se evalúen y se tomen en cuenta tanto en la construcción de obras como en el aprovechamiento de recursos hidráulicos.

Este ensayo tiene como objetivo mostrar las características primordiales de una nueva fuente primaria para los estudiosos de la administración, el uso social y aspectos técnicos de los recursos hidráulicos en México, que se encuentra en el recién constituido Archivo Histórico del Agua.

* Agradecemos el dictamen efectuado a este artículo por la maestra Carmen Nava Nava y el doctor Luis Aboites Aguilar, asimismo las sugerencias hechas por la maestra Guillermina del Valle Pavón. 
108 Maria Teresa Pacho Rodríguez y Gabriel Rivera García

\section{CREACIÓN DEL ARCHIVO HistóRICO DEL AGUA Y RESCATE DEL ACERVO DE LA COMISIÓN DEL Río GRIJALVA.}

A principios de 1994, en su búsqueda de fuentes primarias para la investigación de los usos sociales y aprovechamiento del agua en México, el doctor Luis Aboites encontró en varios estados de la república mexicana acervos documentales con este tipo de información, por lo que se propuso su rescate y organización. El proyecto fue apoyado por el Centro de Investigaciones y Estudios Superiores en Antropología Social (CIESAS) y por la Comisión Nacional del Agua (CNA), instituciones que aportaron recursos humanos y materiales para la constitución del Archivo Histórico del Agua (AHA).

En primera instancia, estas instituciones se orientaron al rescate de diversos archivos relacionados con las actividades que el gobierno federal realizó, a través de varias dependencias descentralizadas, para la construcción y aprovechamiento de los recursos hidráulicos. Su principal objetivo fue la recuperación de fuentes primarias para el estudio y análisis de los organismos responsables de la adecuada utilización del agua.

En la primera etapa se emprendieron trabajos de rescate en los estados de Sonora, Morelos, Chihuahua y Tabasco, en donde funcionaron diversas comisiones que realizaban trabajos directamente relacionados con la optimización del uso del agua. En la ciudad de Cárdenas, Tabasco, se encontró una enorme y semideteriorada montaña de documentos que pertenecieron a la Secretaria de Agricultura y Recursos Hidráulicos (SARH), y en particular a la entidad descentralizada conocida como Comisión del Río Grijalva. Dada la importancia de esta fuente documental se inició su rescate y traslado al $\mathrm{AHA}$, donde se encuentra en una primera fase de clasificación por series. En cuanto concluya su ordenamiento servirá como valiosa fuente a los investigadores dedicados a los temas relacionados con los usos del agua.

Para comprender la importancia de este Archivo Histórico, en primer término daremos los antecedentes de la creación y forma en que desarrolló sus trabajos la Comisión del Río Grijalva.

\section{CREACIÓN DE LA COMISIÓN DEL RÍo GRIJALVA}

El 27 de junio de 1951, por decreto presidencial de Miguel Alemán, se instituyó la Comisión del Río Grijalva, dependiente de la Secretaria de Recursos Hidráulicos. Para su creación se tomaron en cuenta las diversas actividades que el gobierno federal tenía en su programa de construcción, el cual incluía los trabajos y obras necesarios para el desarrollo integral de una parte de la cuenca del río Grijalva en 
el territorio mexicano, que cubría una extensión inicial de 49900 kilómetros cuadrados y abarcaba las zonas de los estados de Tabasco, Chiapas y Oaxaca.

En México es sumamente irregular la distribución de los recursos hidráulicos y la precipitación pluvial es muy variable. La república mexicana se divide, de acuerdo con los factores de precipitación y temperatura, en tres zonas que son: desértica y árida, que se encuentra en $31 \%$ del territorio; semiárida en $33 \%$, y subhúmeda y húmeda en otro $33 \%$. En las regiones áridas se requieren obras de almacenamiento para el riego de tierras. En las zonas húmedas, en cambio, se efectúan obras de control de corrientes, las cuales sirven en la producción agrícola y son fuentes para la generación de energía eléctrica.

En la exposición de motivos para este acuerdo se considera que el río Grijalva constituye una seria y constante amenaza por la impetuosidad de sus avenidas y porque se desborda con frecuencia e inunda enormes extensiones, con perjuicios materiales y pérdida de vidas, por lo que se deben estudiar y construir obras de defensa en esa región, consistentes en presas de almacenamiento para el control de las avenidas y encauzamiento de corrientes que se harán en forma armónica, y otras de aprovechamiento que obedezcan a una cuidadosa planeación.

También indica que hay otro factor que se opone al progreso y desarrollo de la región, y es la insalubridad de muchos lugares, debido al clima y, en especial, a la falta de desagüe natural para la eliminación rápida de las aguas sobrantes y perjudiciales, por lo que se deben realizar labores que recomienda la ingeniería sanitaria para estos casos. Propone la construcción de plantas para la utilización de la cuenca, una de las más importantes del país, que permitan fomentar la agricultura y aprovechar los grandes volúmenes de agua y sus caídas en la generación de energía eléctrica.

Considera que el auge agrícola y la producción de energía eléctrica en grandes plantas, serán las bases para el desarrollo industrial de la zona y se crearán factores conexos tales como nuevos centros de población y aumento de los existentes, vías de comunicación en los tramos navegables de los ríos, puertos fluviales, marítimos y aéreos; carreteras; ferrocarriles y líneas telegráficas.

Para los aspectos de defensa y aprovechamiento de las aguas del río Grijalva y para el desarrollo de las actividades conexas se requiere de trabajos y obras de diversa índole, que se agrupan en:

1. Fundamentales o de defensa, como son las presas de control, las de encauzamiento y las de drenaje. De aprovechamiento de riego y de desarrollo de energía, como son la presas de almacenamiento, presas de derivación, canales de riego y de drenaje, canales de fuerza y plantas hidroeléctricas. De ingeniería sanitaria, como son la de abastecimiento de agua potable y campañas contra plagas. 
2. Conexos, tales como vías de comunicación que comprenden puertos, vías navegables, carreteras, ferrocarriles, telégrafos, teléfonos así como trabajos de fomento agrícola e industrial y los que atañen a centros de población.

Para lograr avances deberán observarse lineamientos y un presupuesto determinado. Coordinar actividades a través de un organismo directivo con atribuciones definidas cuya acción sea técnica y administrativa y se someterá a juntas intersecretariales y con presupuesto independiente.

Por la importancia y variedad de los trabajos relativos a la defensa de ríos, obras de riego e ingeniería sanitaria y las conexas, se justifica que la Secretaría de Recursos Hidráulicos realice estas funciones, pero aun dentro de esta Secretaría no se lograría la unidad requerida para su ejecución, por lo que se considera conveniente la creación de un organismo descentralizado que atienda las sugerencias de las secretarías y aproveche su cooperación para realizar sus funciones, con facultades para resolver y libertad en el manejo de sus fondos. Por estos motivos se crea la Comisión del Río Grijalva, como organismo técnico administrativo con facultades para la planeación, elaboración de proyectos y construcción de todas las obras de defensa, de aprovechamiento en riego, desarrollo de energía y de ingeniería sanitaria. Incluye también las obras conexas citadas arriba. ${ }^{1}$

\section{UBICACIÓN Y PROGRAMA DE TRABAJO DE LA COMISIÓN DEL RÍO GRIJALVA}

La Comisión del Río Grijalva tuvo un proyecto de desarrollo hidráulico que abarcó los estados de Tabasco, Chiapas y Oaxaca. No obstante, cabe mencionar que la cuenca Grijalva-Usumacinta tiene su origen en la nación guatemalteca, y su sistema hidrológico abarca, además de los estados mencionados, pequeñas porciones de Campeche y Veracruz. ${ }^{2}$

El proyecto original tenía como meta el aprovechamiento de los recursos agropecuarios de la región, para lo cual se dio a la tarea de crear infraestructura que permitiera utilizar los recursos provenientes del sistema hidrológico del Grijalva y elaboró estudios y obras de diversa índole.

La Comisión tuvo tres etapas de trabajo: la primera abarca de 1951 a 1959 y en ella se inician formalmente los estudios de la Cuenca Grijalva-Usumacinta, para conocer sus corrientes, marcar prioridades de ejecución de obras y definir

1 Diario Oficial de la Federación, miércoles 29 de agosto de 1951, pp. 4-6

2 "Qué es la Comisión del Río Grijalva?", en Manual de bienvenida de la Unidad de Organización y Métodos de la Comisión del Río Grijalva, Secretaría de Recursos Hidráulicos, Cárdenas, Tabasco, 1976; Archivo Histórico del Agua: Archivo Histórico de la Comisión del Río Grijalva. Serie: Vocalía Ejecutiva, Subserie: Unidad de Organización y Métodos. 
sitios posibles para almacenamiento de aguas; asimismo se proyectan y realizan obras relacionadas con el abastecimiento de agua potable. ${ }^{3}$

En la segunda, de 1959 a 1972, destaca la construcción de caminos y de la presa Netzahualcóyotl; además, la Comisión Federal de Electricidad realizó estudios en la presa La Angostura, del río Grande de Chiapas, con el objetivo de aprovechar su cauce para la generación de energía eléctrica. También hay estudios de las presas Chicoasén y Reñites. En esta etapa sobresale la creación de ejidos para impulsar la producción agrícola intensiva, base del denominado Proyecto El Limón, que posteriormente derivó en el Plan de La Chontalpa, proyecto piloto para desarrollar 300 000 hectáreas. Para su ejecución se llevó a cabo la reestructuración de la tenencia de la tierra, sustentada en la expropiación agrícola, el reacomodo ejidal y la construcción de la presa Netzahualcóyotl. Los fondos para financiamiento de obras e indemnizaciones provenían del erario federal y de préstamos directos del Banco Interamericano de Desarrollo. El proyecto de La Chontalpa surge al iniciar la década de los sesenta, previendo la necesidad de incrementar la producción agrícola, que pueda satisfacer los requerimientos de la población para 1980.

En la última etapa, de 1972 a 1985, se proyectó e inició el Plan BalancánTenosique, semejante al de La Chontalpa, cuya meta era integrar 115000 hectáreas al desarrollo agrícola. Se crea también el Distrito 3 de Tabasco dedicado a la acuacultura, y se construyen drenes y cauces para facilitar el transporte pluvial. ${ }^{4}$

\section{ANTECEDEnTES de LA Comisión NACIONAL del Agua}

El agua tiene espacios geográficos y temporales que determinan su aprovechamiento interrelacionándose con otros recursos naturales. Su disponibilidad depende de su ubicación y de la capacidad que se desarrolle para hacerla llegar a las diferentes regiones y usuarios. En algunas ocasiones está disponible mediante mínimo esfuerzo, pero la mayoría de las veces hay que efectuar obras, desde las muy sencillas hasta las de sofisticada ingeniería, para poder obtenerla y utilizarla.

A partir del triunfo del movimiento revolucionario de 1910, las fuerzas políticas del país establecieron un pacto político para que el gobierno nacional tuviera una vida institucional estable, que le permitiera impulsar programas de desarrollo

3 Uno de los trabajos realizados con anterioridad para facilitar recursos materiales a la región es el de Higgins Industries, Inc.,Estudio sobre México. Economía-Transporte-Navegación, Banco de México, México, 1949, t. I y II (Monografías Industriales del Banco de México, SA).

4 Memoria de la Comisión del Río Grijalva 1951-1987, Secretaría de Agricultura y Recursos Hidráulicos, México, 1987 
social y económico. Entre éstos sobresale el impulso a la producción agrícola que requería, desde luego, aprovechar óptimamente los recursos hidráulicos.

\section{DEPENDENCIAS ADMINISTRATIVAS DE LOS RECURSOS HDRÁULICOS}

Secretaría de Fomento, Colonización e Industria, Dirección de Aguas: Departamento de Concesiones, Departamento de Irrigación.

1921. Secretaría de Agricultura y Fomento, Dirección de Irrigación.

1923. Secretaría de Agricultura y Fomento, Dirección de Aguas: Departamento de Reglamentación e Irrigación.

1926. Secretaría de Agricultura y Fomento, Comisión Nacional de Irrigación.

1936. Dirección de Pequeña Irrigación.

1947. Comisión Nacional de Irrigación: Programa Nacional de Irrigación, Secretaría de Agricultura y Ganadería, Secretaría de Recursos Hidráulicos.

\section{La Secretaría de Recursos HidRátlicos y las Comisiones PARA \\ EL APROVECHAMIENTO DEL AGUA}

En el régimen de Miguel Alemán, las labores desarrolladas por la Comisión se incrementaron, lo que condujo a la administración federal a crear dos secretarías que asumieran las funciones de la Comisión. Esas instancias fueron la Secretaría de Agricultura y Ganadería y la Secretaría de Recursos Hidráulicos, que iniciaron sus actividades en enero de 1947. Con excepción de lo relacionado con los distritos de riego y de suelos que por decreto presidencial pasaron a la Secretaría de Agricultura y Ganadería, la Secretaría de Recursos Hidráulicos asumió las facultades de la Comisión de Irrigación y también el aprovechamiento de los recursos hidráulicos para la generación de energía eléctrica y el abastecimiento de agua potable. Posteriormente mediante acuerdos y decretos presidenciales, absorbió también a las direcciones de Aguas, Ingeniería Sanitaria y de Obras Hidráulicas, que dependían, respectivamente, de las secretarías de Agricultura, de Salubridad y Asistencia y de Comunicaciones y Obras Públicas.

La diversidad y complejidad de las tareas asumidas por la nueva secretaría llevaron al gobierno federal a la creación de varias comisiones ejecutivas encargadas de programas de desarrollo hidráulico específico en distintas regiones del país, como las del Río Balsas, del Fuerte, Papaloapan, Grijalva, Aguas del Valle de México, de la Cuenca del Río Pánuco, de Aprovechamiento de Aguas Salinas y del Lago de Texcoco. 
Las comisiones eran organismos ejecutivos que concentraban tareas de diversas secretarías de Estado, programando detalladamente sus actividades para promover el desarrollo integral o parcial de un territorio, definido como una unidad hidrológica natural. La programación por cuenca partió de un conjunto de obras de infraestructura hidráulica, esencialmente para el control de las inundaciones, desarrollando obras de orden social y económico. El modelo en que se basó esta estrategia fue el de la Tennessee Valley Authority, ${ }^{5}$ creada en Estados Unidos en 1933, como medio para enfrentar los efectos de la depresión económica de 1929 en regiones de recursos degradados. ${ }^{6}$

Después de esta breve revisión de los antecedentes de la Comisión del Río Grijalva, que surgió como una entidad que resolvería los problemas de utilización de recursos hidráulicos, de una región con potencial para establecer modelos agrícolas e industriales que ayudarían al desarrollo de México, presentamos el Archivo Histórico de la Comisión del Río Grijalva, en donde se reflejan sus actividades.

\section{El ARChivo Histórico de LA Comisión DEL Río Grijalva}

El Archivo Histórico de la Comisión del Río Grijalva está contenido en 344 cajas de $1.10 \mathrm{~m}$ lineales identificados en tres niveles, que equivalen a 378.40 metros de documentación inexplorada. La documentación está clasificada en 12 series, 65 subseries y 60 subsubseries que corresponden al organigrama que tenía la Comisión en $1985 .{ }^{7}$ En este organigrama y en el del Plan de La Chontalpa se fue insertando el material, que se separó por temas, y que tenía una correspondencia natural. Para explicar esta correspondencia damos los siguientes ejemplos, primero en forma gráfica y posteriormente separando con diagonales cada una de las series, subseries y subsubseries del Archivo, y a continuación incluimos algunos ejemplos de los documentos respectivos señalados con guión. En algunos casos, insertamos después una breve explicación del contenido informativo de la serie.

\section{ORGANIZACIÓN}

Vocalía Ejecutiva/Unidad de Organización y Métodos/Manuales de Organización. -Información sobre la organización y funcionamiento: Promotora del Complejo agropecuario Balancán- Tenosique. Proyecto y organización.

5 Véase Wilmon Henry Droze, Presas gigantescas yaguas apacibles, Limusa Wiley, México, 1966.

6 Véase Blanca Torres, Historia de la revolución mexicana. 1940-1956. Hacia una utopía industrial, El Colegio de México, México, 1984, p. 71

7Esta propuesta de organización la hizo el equipo que rescató y ordenó en su primera fase el Archivo Histórico de la Comisión del Río Grijalva. 


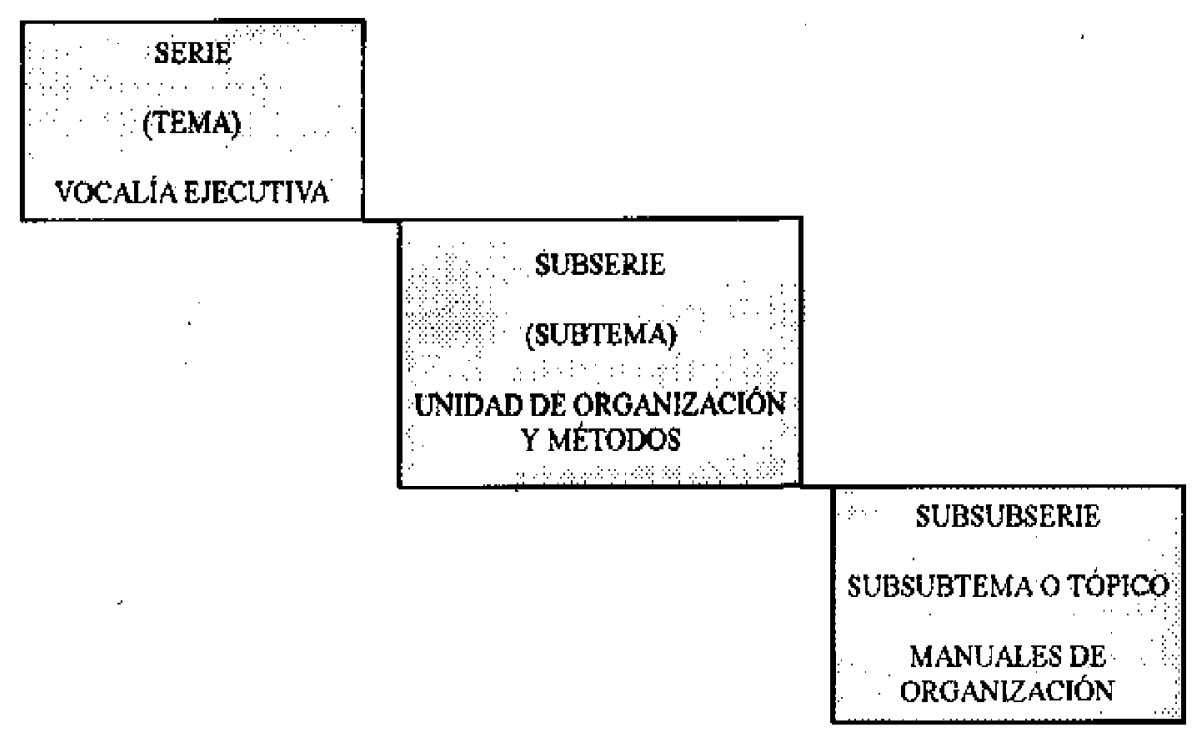

Comisión. Promotora del complejo agroindustrial de La Chontalpa/Dirección de Catastro y Servicios Sociales/Catastro, avalúos e indemnizaciones.

-Sobre la distribución de las tierras, cultivos y población de la zona. Comisión Promotora del complejo agroindustrial de La Chontalpa/Titulación de parcelas y viviendas/Predios certificados agrarios provisionales.

-Redistribución de la población y la posesión de la tierra y los cultivos. Dirección de operación y conservación/Plan Chontalpa préstamos del Banco Interamericano de Desarrollo.

-Lo referente a planes y solicitudes de crédito.

Dirección de operación y conservación/Jefatura de operación y conservación de obras hidráulicas.

-Sobre operación y conservación de obras.

Dirección de Investigaciones agrícolas y ganaderas/Escuela Técnico Agrícola! Colegio Superior de Agricultura Tropical.

-Contiene lo relativo a la liquidación de esta institución.

Gerencia del Bajo Grijalva (Malpaso)/Residencia de operación presa Netzahualcóyot1/Presa Netzahualcóyotl.

-Sobre los proyectos y funcionamiento de la presa.

Gerencia del Bajo Grijalva (Malpaso)/Residencia de Construcción/Constructora Raudales.

-Acerca de la empresa creada para la construcción de la presa. Proyecto Piloto Limón.

-Presupuestos y planes del mismo. 
También hay información sobre los distritos de riego; las obras de alcantarillado, drenaje y dotación de agua potable ejecutadas en Chiapas y Tabasco; residencias de estudio del cauce Samaria-Golfo de México y expedientes del personal que laboró en la Comisión del Río Grijalva.8

A continuación se ejemplifican los contenidos de algunos expedientes: Indemnizaciones y catastro: Tienen un número de afectación, son expedientes emitidos por la Sección de Recursos Hidráulicos de la Comisión del Grijalva y del Plan de La Chontalpa; contienen: clave de expediente, fecha de recibo, número de expediente, nombre del afectado, fotografía del afectado y recibo con los datos de clasificación. Continúa el expediente con el dictamen y la resolución. El dictamen nos da el régimen del afectado y la forma en la que queda. Evalúa primero los cultivos afectados y después la construcción en el predio afectado. Dentro de la evaluación de cultivos se indica: el tipo, la cantidad, la unidad de medida, el precio unitario y el total. En cuanto a las construcciones, el número, para qué se utiliza, las dimensiones y características de construcción. Termina el expediente con un estudio catastral con la ubicación del predio descrita por croquis o plano y la hoja de los datos personales del afectado.

Este tipo de expediente refleja la forma y condiciones en que se dieron las afectaciones y los pormenores de la forma y cantidad de pagos efectuados en esta zona.

Avalios. Comienza con el número de expediente, la fecha y el importe. Continúa con la fundamentación legal para llevar a cabo el avalúo. Establece el nombre del ejidatario, la unidad agropecuaria, fecha de inspección y la conclusión con los datos personales del interesado. Estos expedientes incluyen fotografías de la vivienda. En algunos hay censo de población para apoyar la demanda de avalúo y aparecen mujeres ejidatarias.

Personal. Son contratos del personal por 170 días, con el nombre del contratado y el grupo al que pertenece. Trae cláusulas y certificado médico.

Personal. Expedientes de contrataciones temporales; en algunos casos aparece el personal que se va a contratar. Algunos expedientes tienen testamentos, cambios de salarios, vacaciones, informes, nóminas, facturas y fotografías de los contratados.

Como se puede apreciar, el Archivo Histórico de la Comisión del Río Grijalva, institución sobre la que casi no hay estudios efectuados, ofrece al investigador una diversidad de temas relacionados no sólo con los usos sociales y el aprovechamiento del agua, sino también con el manejo que el Estado da a los recursos

8 Esta división es una propuesta de cuadro clasificador para ordenar el Archivo Histórico de la Comisión del Río Grijalva, y en donde la serie corresponde a la oficina o departamento que llevaba a cabo tareas especificas, y la subserie - la subsubserie al material que se separó por tema, respetando las características tipológicas que corresponden a cada entidad generadora. 
hidráulicos dentro de sus políticas para el fomento de la producción agrícola, y con la construcción de infraestructura en la zona de Tabasco y Chiapas para establecer zonas de cultivo, vías de comunicación para el comercio y obras para la generación de energía eléctrica. Asimismo, aborda la historia de la expropiación y reparto de tierras en la zona; del reacomodo de ejidatarios y poblados; de la implantación de un modelo de producción agrícola que, se creía, iba a satisfacer los requerimientos alimentarios del país, y de la creación de un modelo agrícola

industrial que daría empleo a los habitantes de estos estados. También es una fuente para el estudio financiero y técnico de las obras para el almacenamiento de agua, del cambio de curso de ríos, de la dotación de drenaje yagua potable a diversos poblados y de los trabajadores que hicieron posibles estas obras. 